\title{
SUBSEQUENCES AND CATEGORY
}

\author{
ROBERT R. KALLMAN
}

(Received 21 July 1998)

\begin{abstract}
If a sequence of functions diverges almost everywhere, then the set of subsequences which diverge almost everywhere is a residual set of subsequences.
\end{abstract}

Keywords and phrases. Baire category, measure, measurable functions, sequences.

1991 Mathematics Subject Classification. 28A20, 54E52.

1. Introduction. In [1], Bilyeu, Lewis, and Kallman proved a general theorem about rearrangements of a series of Banach space valued functions. This theorem settled a question on rearrangements of Fourier series posed by Kac and Zygmund. Kallman [3] proved an analog of this theorem for subseries of a series of Banach space valued functions. The purpose of this paper is to complete the cycle of these ideas by proving an analogous theorem (Theorem 1.1) for subsequences of a sequence of Banach space valued functions. Theorem 1.1 does not seem to follow directly from results of [1] or [3]. Other than [1, 3], the only precedent for Theorem 1.1 seems to be a paper [7] on subsequences of a sequence of complex numbers.

Let $S$ be the set of all sequences $s=\left(s_{1}, s_{2}, \ldots\right)$, where $1 \leq s_{1}<s_{2}<\cdots$ is a strictly increasing sequence of positive integers. $S$ is a closed subset of the countable product of the positive integers, and so $S$ is a complete separable metric space. Given any sequence of objects $a_{1}, a_{2}, \ldots$, one can identify the set of its subsequences both as a set and as a topological space with $S$. In this context, it is natural to identify a collection of subsequences with a subset of $S$ and ask if it is first category, second category, or residual ([5] or [6]). Define an equivalence relation $\sim$ on $S$ as follows: if $s, t \in S$, then $s \sim t$ if and only if $s_{n}=t_{n}$ for all sufficiently large $n$. Intuitively this states that $s \sim t$ if and only if $s$ and $t$ agree from some point on. It is simple to check that any nonempty subset of $S$ which is saturated with respect to $\sim$ is dense.

The main result of this paper is the following theorem, which is proved in Section 2.

THEOREM 1.1. Let $(X, \mu)$ be a regular locally compact $\sigma$-finite measure space, $Z$ a separable Banach space, and $f_{n}: X \mapsto Z$ a sequence of Borel measurable functions. Suppose that the sequence $f_{n}(x)$ diverges for $\mu$-a.e., $x \in X$. Then $\left[s \in S \mid f_{s_{n}}(x)\right.$ diverges for $\mu$-a.e. $x \in X]$ is a residual set in $S$.

Just as in [1, 3], this measure-category result has a category-category analog which is discussed in Section 3.

2. Proof of Theorem 1.1. The following special case of Theorem 1.1 will be proved first. 
LEMMA 2.1. Let $K$ be a compact Hausdorff space, $Z$ a Banach space, and $f_{n}: K \mapsto Z$ a sequence of continuous functions, and $\delta>0$. Suppose that for every $x \in K$ and positive integer $N$, there exists a pair of integers $n=n(x, N)$ and $m=m(x, N)$ so that $N \leq n \leq m$ and $\left\|f_{m}(x)-f_{n}(x)\right\|>\delta$. Then $\left[s \in S \mid f_{s_{n}}(x)\right.$ diverges for every $\left.x \in K\right]$ is a residual set in $S$.

Proof. If $m, n$ is a pair of integers such that $1 \leq n \leq m$ and $s \in S$, let $g_{s, m, n}: K \mapsto$ $[0,+\infty)$ be defined by $g_{s, m, n}(x)=\left\|f_{s_{m}}(x)-f_{s_{n}}(x)\right\| . g_{s, m, n}$ is continuous. Consider

$$
A=\bigcap_{N \geq 1} \bigcup_{N \leq n_{1} \leq m_{1}, \ldots, N \leq n_{p} \leq m_{p}}\left[s \in S \mid \cup_{1 \leq i \leq p} \mathcal{g}_{s, m_{i}, n_{i}}^{-1}((\delta,+\infty))=K\right] .
$$

Fix $1 \leq n \leq m$ and $s \in S$. Then $V=\left[t \in S \mid t_{m}=s_{m}\right.$ and $\left.t_{n}=s_{n}\right]$ is an open neighborhood of $s$ in $S$. Hence, if $t \in V$, then $g_{t, m, n}=g_{s, m, n}$. This in turn implies that $A$ is a $G_{\delta}$ subset of $S$. Furthermore, $A$ is saturated with respect to the equivalence relation $\sim$ and therefore is a dense $G_{\delta}$ if it is nonempty.

$A$ is nonempty since $t=(1,2,3, \ldots)$ is in $A$. To see this, fix $N \geq 1$. For $N \leq n \leq m$, let $U(m, n)=g_{t, m, n}^{-1}((\delta,+\infty))$. Note that the collection $\{U(m, n)\}_{N \leq n \leq m}$ is an open covering of $K$ by hypothesis and so has a finite subcover, say $U\left(m_{1}, n_{1}\right), \ldots, U\left(m_{p}, n_{p}\right)$. One easily concludes from this that $t \in A$.

Finally, note that the Cauchy criterion for convergence implies that if $s \in A$, then $f_{s_{n}}(x)$ diverges for every $x \in K$. Hence, $A \subseteq\left[s \in S \mid f_{s_{n}}(x)\right.$ diverges for every $\left.x \in K\right]$. This proves Lemma 2.1.

Proof OF Theorem 1.1. We may assume that $\mu$ is a probability measure since $\mu$ is $\sigma$-finite. If $q \geq 1$, let

$$
D_{q}=\bigcap_{N \geq 1} \bigcup_{N \leq n \leq m}\left[x \in X \mid\left\|f_{m}(x)-f_{n}(x)\right\|>\frac{1}{q}\right] .
$$

Each $D_{q}$ is a Borel subset of $X, D_{q} \subseteq D_{q+1}$, and the Cauchy criterion for convergence implies that $\cup_{q \geq 1} D_{q}=\left[x \in X \mid f_{n}(x)\right.$ diverges $] . \mu\left(\cup_{q \geq 1} D_{q}\right)=1$ by assumption. Use a vector-valued version of Lusin's Theorem [2] to choose, for each $q$, a compact subset $K_{q}$ of $D_{q}$ so that each $f_{n} \mid K_{q}$ is continuous and $\mu\left(D_{q}-K_{q}\right)<1 / q . R_{q}=\left[s \in S \mid f_{s_{n}}(x)\right.$ diverges for every $\left.x \in K_{q}\right]$ is a residual subset of $S$ by Lemma 2.1. Hence, $R=\cap_{q \geq 1} R_{q}$ is a residual set in $S$ and is contained in $\left[s \in S \mid f_{s_{n}}(x)\right.$ diverges for $\mu$-a.e., $x \in X$ ] since $\mu\left(\cup_{q \geq 1} K_{q}\right)=1$. This proves Theorem 1.1.

3. Sequences of functions with the Baire property. Theorem 1.1 may be regarded as a measure-category result. The purpose of this section is to prove a categorycategory analog of Theorem 1.1 (cf. [1, Thm. 1.2] and [3, Thm. 3.1]).

Let $X$ be a Polish space. $A$ subset of $X$ is said to have the Baire property if there exists an open set $U$ in $X$ so that $A \triangle U$ is first category. The collection of all subsets of $X$ with the Baire property is a $\sigma$-algebra which includes the analytic sets in $X$. Let $Z$ be any other Polish space. A function $f: X \mapsto Z$ is said to have the Baire property if $U$ open in $Z$ implies that $f^{-1}(U)$ has the Baire property in $X$. Any Borel function $f: X \mapsto Z$ is a function with the Baire property. See [4, 5] or [6] for a thorough discussion of this circle of ideas. The following theorem is then a category-category analog of Theorem 1.1. 
Theorem 3.1. Let $X$ be a Polish space, $Z$ a separable Banach space, and $f_{n}: X \mapsto Z$ a sequence of functions with the Baire property. Suppose that $\left[x \in X \mid f_{n}(x)\right.$ diverges] is a residual subset of $X$. Then $\left[s \in S \mid f_{s_{n}}(x)\right.$ diverges on a residual subset of $\left.X\right]$ is a residual subset of $S$.

The following proposition, of independent interest, is needed to prove Theorem 3.1.

Proposition 3.2. Let $Z$ be a Banach space and let $\left\{z_{n}\right\}_{n \geq 1}$ be a sequence in $Z$. Let $A=\left[s \in S \mid z_{s_{n}}\right.$ converges $]$. Then either $A=S$ or $A$ is of first category in $S$.

Proof. For $k \geq 1$ define

$$
B_{k}=\bigcap_{N \geq 1} \bigcup_{N \leq n \leq m}\left[s \in S \mid\left\|z_{s_{m}}-z_{s_{n}}\right\|>\frac{1}{k}\right] .
$$

Note that $B_{k} \subseteq B_{k+1}$. Each set in square brackets is open in $S$. Hence, this formula shows that $B_{k}$ is a $G_{\delta} . B_{k}$ is dense if it is nonempty since it is saturated with respect to the equivalence relation $\sim$. Therefore, $B_{k}$ is a residual set in $S$ if it is nonempty since any dense $G_{\delta}$ is residual.

The Cauchy criterion for convergence implies that $A^{c}=\cup_{k \geq 1} B_{k}$. Hence, either $A=$ $S$ or $A^{c}$ is residual in $S$; or either $A=S$ or $A$ is of first category in $S$. This proves Proposition 3.2.

Proof OF Theorem 3.1. Check that the mapping $(x, s) \mapsto f_{s_{n}}(x), X \times S \mapsto Z$, is a function with the Baire property for every $n \geq 1$. Hence,

$$
B=\left[(x, s) \mid f_{s_{n}}(x) \text { diverges }\right]=\bigcup_{k \geq 1} \bigcap_{N \geq 1} \bigcup_{N \leq n \leq m}\left[(x, s) \mid\left\|f_{s_{m}}(x)-f_{s_{n}}(x)\right\|>\frac{1}{k}\right]
$$

is a subset of $X \times S$ with the Baire property. For each $x \in X$, let $B_{x}^{c}$ be the projection of $B^{c} \cap((x) \times S)$ onto $S$. The hypotheses of Theorem 3.1 plus Proposition 3.2 imply that each $B_{x}^{c}$ is a first category subset of $S$, except for a first category set of $x$ 's. But then $B^{c}$ is itself a first category subset of $X \times S$ [6, Thm. 15.4] and so $B_{s}^{c}$, the projection of $B^{c} \cap(X \times(s))$ onto $X$, is a first category subset of $X$, except for a first category set of $s$ 's (Theorem of Kuratowski-Ulam, [6, Thm. 15.1]). Hence, $B_{s}$, the projection of $B \cap(X \times(s))$ onto $X$, is a residual subset of $X$ for all except a first category set of $s$ 's. This proves Theorem 3.1.

\section{REFERENCES}

[1] R. G. Bilyeu, R. R. Kallman, and P. W. Lewis, Rearrangements and category, Pacific J. Math. 121 (1986), no. 1, 41-46. MR 87j:42019. Zbl 574.42007.

[2] J. Diestel and J. J. Uhl, Jr., Vector measures, Mathematical Surveys, no. 15, American Mathematical Society, Providence, RI, 1977. MR 56 12216. Zbl 369.46039.

[3] R. R. Kallman, Subseries and category, J. Math. Anal. Appl. 132 (1988), no. 1, 234-237. MR 89g:46074. Zbl 676.46004.

[4] A. S. Kechris, Classical descriptive set theory, Graduate Texts in Mathematics, vol. 156, Springer-Verlag, New York, 1995. MR 96e:03057. Zbl 819.04002.

[5] K. Kuratowski, Topology. Vol. I., Academic Press, New York, London, 1966. MR 36\#840. Zbl 158.40802.

[6] J. C. Oxtoby, Measure and category, Graduate Texts in Mathematics, vol. 2, Springer-Verlag, New York, Berlin, 1980. MR 81j:28003. Zbl 435.28011. 
[7] M. B. Rao, K. P. S. B. Rao, and B. V. Rao, Remarks on subsequences, subseries and rearrangements, Proc. Amer. Math. Soc. 67 (1977), no. 2, 293-296. MR 5812068. Zbl 412.40001.

KALLman: Department of MATHEMatics, University of NoRTh TeXas, P. O. BOX 305118, DENTON, TEXAS 76203-5118, USA 


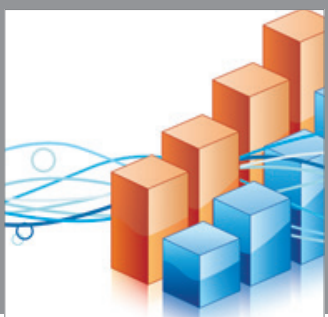

Advances in

Operations Research

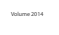

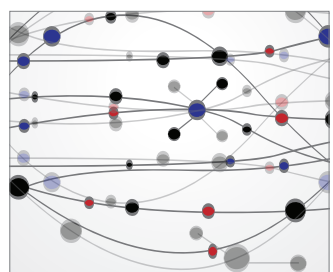

\section{The Scientific} World Journal
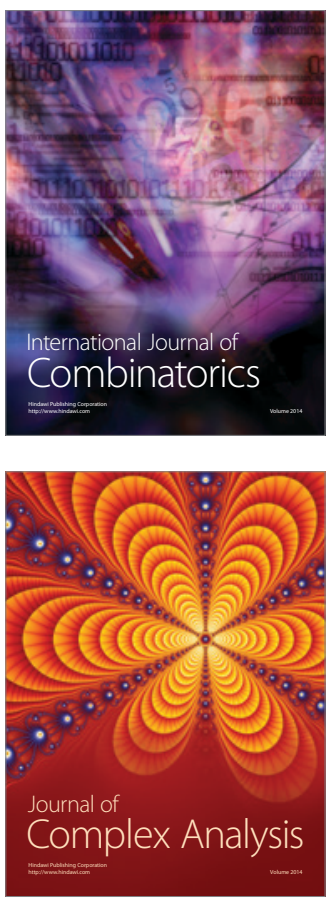

International Journal of

Mathematics and

Mathematical

Sciences
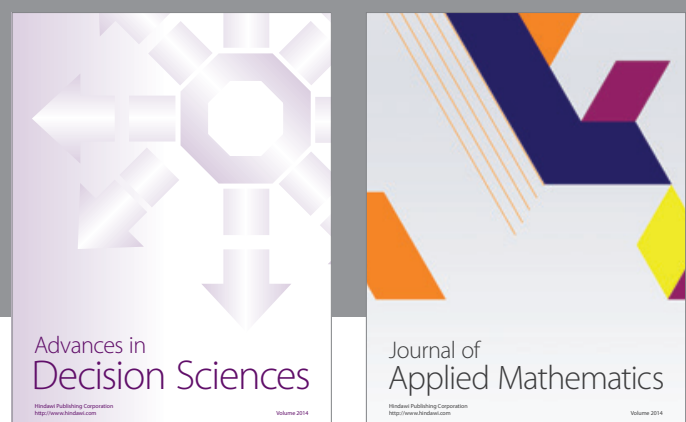

Journal of

Applied Mathematics
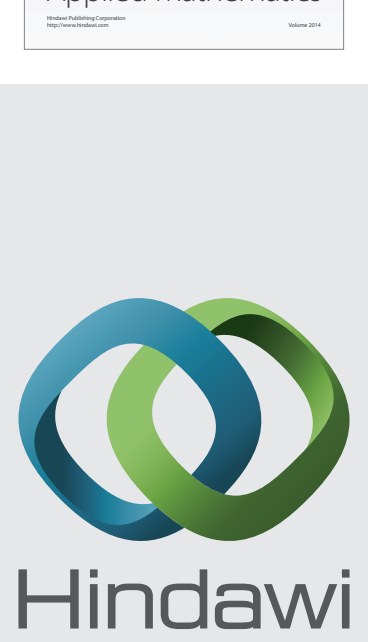

Submit your manuscripts at http://www.hindawi.com
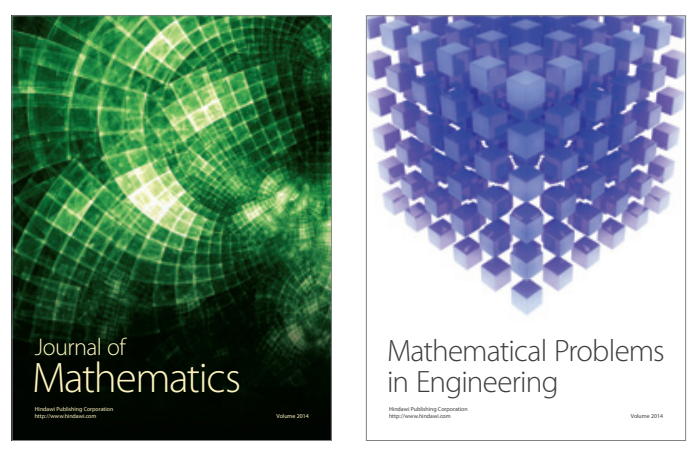

Mathematical Problems in Engineering
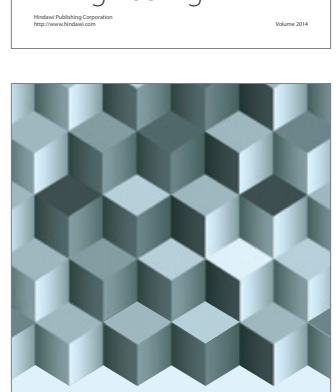

Journal of

Function Spaces
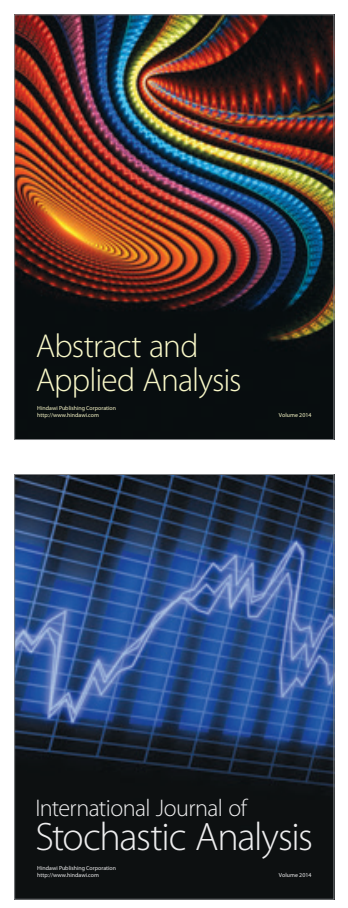

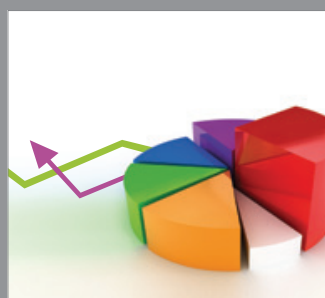

ournal of

Probability and Statistics

Promensencen
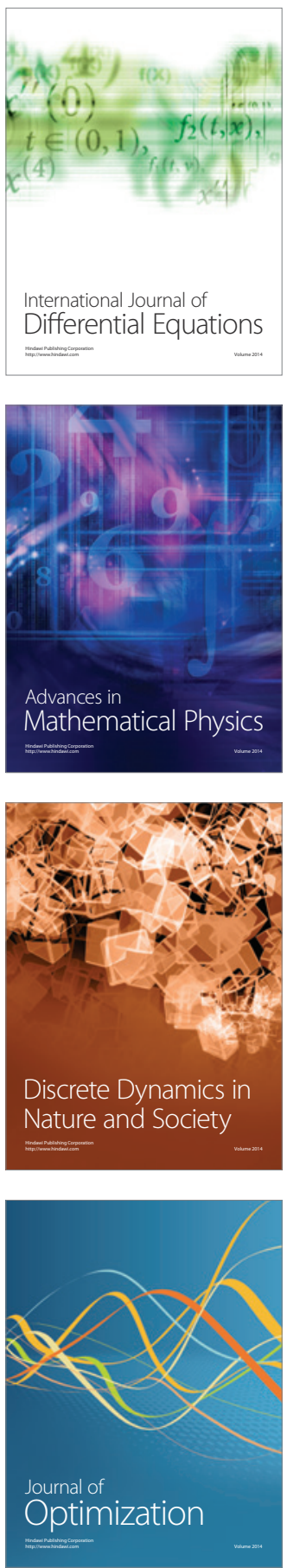